\title{
Restoring tactile and proprioceptive sensation through a brain interface
}

\author{
Gregg A. Tabot ${ }^{1}$, Sung Shin Kim ${ }^{2}$, Jeremy E. Winberry ${ }^{2}$, and Sliman J. Bensmaia ${ }^{1,2^{*}}$ \\ ${ }^{1}$ Committee on Computational Neuroscience, University of Chicago, Chicago, IL \\ ${ }^{2}$ Department of Organismal Biology and Anatomy, University of Chicago, Chicago, IL \\ *Corresponding author:
}

Tel: 773.834 .5204

Fax: 773.702 .0037

Address: 1027 E $57^{\text {th }}$ street, Chicago, IL, 60637

Acknowledgments: This work supported by Defense Advanced Research Projects Agency under contract N6601-10-C-4056, by the National Science Foundation Grant DGE-0903637, by the National Institutes of Health Grant RO1 NS08285, and by the Chicago Biological Consortium Grant c-049. 
Somatosensation plays a critical role in the dexterous manipulation of objects, in emotional communication, and in the embodiment of our limbs. For upper-limb neuroprostheses to be adopted by prospective users, prosthetic limbs will thus need to provide sensory information about the position of the limb in space and about objects grasped in the hand. One approach to restoring touch and proprioception consists of electrically stimulating neurons in somatosensory cortex in the hopes of eliciting meaningful sensations to support the dexterous use of the hands, promote their embodiment, and perhaps even restore the affective dimension of touch. In this review, we discuss the importance of touch and proprioception in everyday life, then describe approaches to providing artificial somatosensory feedback through intracortical microstimulation (ICMS). We explore the importance of biomimicry - the elicitation of naturalistic patterns of neuronal activation - and that of adaptation - the brain's ability to adapt to novel sensory input, and argue that both biomimicry and adaptation will play a critical role in the artificial restoration of somatosensation. We also propose that the documented re-organization that occurs after injury does not pose a significant obstacle to brain interfaces. While still at an early stage of development, sensory restoration is a critical step in transitioning upper-limb neuroprostheses from the laboratory to the clinic.

\section{Introduction}

One approach to restoring sensorimotor function to amputees or tetraplegic patients is to equip them with robotic prosthetic arms and have them control these limbs using signals from the motor parts of their brain. To date, a number of patients have been able to control anthropomorphic robotic arms to perform simple motor tasks, such as grasping and moving objects (Collinger et al., 2013; Hochberg et al., 2012). As impressive as these feats are from a scientific and technological standpoint, however, movements generated using these prosthetic limbs are slow and relatively inaccurate; their performance still does not warrant the highly invasive surgery that they would require if they were adopted in the clinic. One important reason for the poor performance is the lack of somatosensory feedback. Indeed, our ability to grasp and manipulate objects is critically dependent on the senses of touch and proprioception. Tactile and proprioceptive signals from the hand convey information about the size, shape, and texture of objects, and indicate when objects are slipping from our grasp (Johansson and Flanagan, 2009). Proprioceptive signals also convey information about the state of our limb and are important to plan and guide movements (Sainburg et al., 1995; Sainburg et al., 1993). Vision is a poor substitute for touch and proprioception as evidenced by the fact that patients with intact motor but impaired somatosensory function struggle to perform activities of daily living, like tying a shoelace or turning a doorknob (Marsden et al., 1984). Touch also plays a critical role in emotional and social communication - we touch the people we care about - and in embodiment - the sense that our body is part of us. Given the importance of somatosensation in intact individuals, a key to improving the performance of prosthetic arms is to incorporate this critical sensory feedback. One approach to restoring somatosensation is to electrically stimulate the somatosensory parts of the brain, specifically primary somatosensory cortex (Figure 1), in the hopes of eliciting meaningful and informative tactile percepts that can then be used not only to improve the dexterity of these limbs, but also to restore the affective component of touch and promote the embodiment of the prosthesis.

\section{Somatosensation and dexterous motor control}


When we grasp and manipulate objects, tactile and proprioceptive signals from our hands convey information about the shape, size, and texture of the objects, and signal when they are slipping from our grasp (Johansson and Flanagan, 2009). A variety of different cutaneous receptors convey information about skin deformations (Bensmaia and Tillery, 2013), and different receptors in the joints, muscles, and skin convey information about the conformation and movements of the limb (Edin, 1990; Edin, 2004). Without these signals, we would frequently crush or drop objects and could not perform dexterous tasks, such as typing on a keyboard or playing a musical instrument. The importance of somatosensory feedback in most activities of daily living is underscored in lesion studies in monkeys, showing that inactivation of S1 results in uncoordinated finger movements and coarse or exaggerated grasp behaviors, presumably to compensate for attenuated sensation (Brochier et al., 1999). In patients with peripheral neuropathies that abolish somatosensory afference while leaving motor efference intact, the loss of proprioception impairs reaching movements, often resulting in inaccurate trajectories (Gordon et al., 1995). Proprioceptive signals are thus required for smooth reaching movements and play a critical role in correcting movement errors.

\section{Touch and affective communication}

The manner in which we touch someone, for example the speed at which we stroke them, can communicate distinct emotions, such as anger, fear, sadness, love and so on, and the accuracy with which this emotional information is transmitted by touch is comparable to that of vocal and facial expression (Hertenstein et al., 2009). At the somatosensory periphery, pleasant touch is in part mediated by a specialized set of afferents, namely C-tactile afferents, that innervate the hairy skin (Löken et al., 2009). Counterparts to these afferents have yet to be found in glabrous skin. Several regions in cortex are associated with the affective and social aspects of touch. Signals related to the pleasantness of touch are found in S1, where activation can be modulated by social context (that is, depending on who does the touching) (Gazzola et al., 2012). A subpopulation of S1 neurons responds to the observation of touch and may support social communication through empathy, a phenomenon dubbed 'mirror-touch synesthesia' (Keysers et al., 2010). The posterior insular cortex receives projections from C-tactile afferents and is thus implicated in the experience of pleasant touch (Björnsdotter et al., 2009). Affective touch is also represented in orbitofrontal cortex, which is generally associated with affective value (Kringelbach, 2005) and responds relatively weakly even to neutral touch (Rolls et al., 2003). Thus, to the extent that S1 activity encodes affective touch and drives activity in other affect-related brain areas, S1 stimulation might be used to restore this critical aspect of somatosensation.

\section{Touch and embodiment}

We experience our limbs as being part of our body; in fact, this embodiment does not necessarily disappear when we lose a limb. Indeed, many amputees experience a phantom limb, the sensation that the missing limb is still there (Ramachandran and Hirstein, 1998). Most patients report that the phantom limb is in a static position and immovable. These sensations can gradually fade over a period of weeks but about $30 \%$ of those who experience phantom limbs do so for years or even decades after limb loss (Sherman, 1989). That the phantom limb is perceived as being static often gives rise to sensations of pain or discomfort, even if the phantom limb is perceived to be in a comfortable resting position. Moving the phantom limb would be sufficient to reduce or eliminate the pain but many amputees are unable to move their phantom limbs even if they try to imagine moving it. The presence of phantom limbs is thought to be caused by activity in somatosensory areas in cortex that used to receive input from the limb and is thus perceptually projected to the limb. One approach to alleviating phantom pain 
is mirror box therapy, in which the amputee places intact and missing limbs in two holes, one on each side of a box partitioned by a doubly-reflective mirror. The reflection of the limb in the mirror is perceived to be in the location of the missing phantom limb. Thus, moving the intact limb creates the illusion that the phantom limb is moving, which can relieve the amputee of the painful sensation that the phantom is stuck in an uncomfortable position.

Because most prostheses do not provide somatosensory feedback, amputees often describe them as alien or liken them to a foreign tool. Despite the fact that they are able to move and manipulate their artificial limb, users do not feel like they have a sense of ownership of the limb, especially in the absence of vision (Wright et al., 1995). This lack of embodiment may in part explain why amputees often reject advanced robotic prostheses in favor of simpler, cable-driven or body-powered ones as the latter provide sensory feedback in the form of tension and resistance (Biddiss et al., 2007). In light of this, promoting the embodiment of a prosthetic limb may be an important goal.

The importance of somatosensory signals in embodiment is most compellingly demonstrated with the Rubber Hand Illusion (Botvinick and Cohen, 1998; Tsakiris and Haggard, 2005), which bears similarities to mirror box therapy. In this paradigm, one of the subject's hands is concealed from view, and a lifesized artificial rubber hand is positioned approximately in its place. The experimenter then simultaneously delivers a tactile stimulus (often strokes from a brush) to the same region of the (hidden) intact hand and the artificial rubber hand. When the subject sees the artificial hand being touched while simultaneously feeling a touch on his or her own hand, the subject begins to embody the artificial hand and perceptually incorporate it as his or her own. The synchrony and congruence of the visual and tactile signals is hypothesized to drive this embodiment. Studies with the rubber hand illusion suggest that the elicitation of cutaneous sensations that are synchronous and congruent with the visual experience of the prosthetic limb touching objects will promote embodiment of the limb, a prediction that is borne out in the data (see below).

\section{Electrical stimulation of S1 results in somatosensory percepts}

Beginning in the 1930s, Wilder Penfield stimulated the surface of the brain in search of the foci of epileptic seizures (Penfield and Boldrey, 1937) and found that stimulating the postcentral gyrus elicited tactile percepts that were localized to specific locations on the body. These electrically-induced percepts usually manifested as numbness or tingling and sometimes pain. The projected location of these sensations varied systematically with the location of S1 stimulation, leading to the creation of the first somatosensory homunculus, an illustration depicting the projected locations of the induced percepts on the body visualized over the parts of S1 which, when stimulated, induce those percepts (Figure 2a). In previous human studies investigating the perceptual consequences of electrically stimulating S1, the evoked percepts were relatively diffuse and consisted primarily of paresthesias (see Johnson et al., 2013, e.g.). Note, however, that these were carried out using stimulating electrodes at the surface of the brain, which produce much more diffuse neuronal activation than do interfaces with penetrating electrodes. Indeed, as described below, animal experiments with penetrating electrodes have yielded more clearly defined percepts.

In a landmark study, Romo and colleagues demonstrated that, not only could tactile percepts be elicited through ICMS in S1, but these percepts could be systematically manipulated by changing the parameters of stimulation (Romo et al., 2000; Romo et al., 1998). Specifically, he trained animals to discriminate the frequency of vibrations delivered to the skin with a vibratory motor. Such periodic skin vibrations elicit periodic responses in S1, the frequency of which matches that of the vibration. Romo's team then had 
the animals perform the same discrimination task based not on mechanical stimulation of the skin but on periodic electrical pulse trains delivered to $\mathrm{S} 1$, thereby mimicking the natural response. Remarkably, the animals were able to perform the frequency discrimination task based on this periodic ICMS with comparable accuracy as with skin vibrations. This study suggested that, if the critical aspects of neuronal activity are replicated, in this case the periodic firing, the corresponding aspect of the percept is elicited, in this case perceived vibrotactile frequency.

\section{Biomimicry}

The most straightforward approach to conveying artificial sensory feedback is to attempt to reproduce naturalistic patterns of neuronal activation through ICMS. Indeed, different neuronal populations in S1 convey different information about the state of the limb and about events impinging on it. Some neurons convey information about the shape of objects grasped in the hand (Bensmaia et al., 2008)(Figure 1b), others convey information about the motion of objects across the skin (Pei et al., 2010; Pei et al., 2011)(Figure 1c), still others convey information about limb position and movements (London and Miller, 2013)(Figure 1d), etc. In principle, then, it would be possible to convey information about limb state and object attributes by exploiting these native neuronal representations. To the extent that naturalistic pattern of activity can be elicited through ICMS, then, the evoked sensations will be verisimilar. An intuitive example of the so-called biomimetic approach is the exploitation of somatotopy to convey information about contact location.

Primary somatosensory cortex is well known to be organized somatotopically as illustrated by the somatosensory homunculus. Indeed, adjacent S1 neurons respond to adjacent body regions, with some discontinuities (between the hand and the face representations, e.g., see Figure 2a). Thus, when an object makes contact with a part of our body, the corresponding part of S1 gets activated. The aforementioned experiments by Penfield suggested that where we feel a touch might be determined by where on the homunculus the activated neurons fall. This conclusion was confirmed in experiments on monkeys, which demonstrated that stimulation through an individual electrode in S1 did indeed result in tactile percepts that were localized to the receptive field of that electrode (Tabot et al., 2013).

Information about what part of the hand is contacting an object is critical for grasping and manipulating it: For example, the thumb and one of the other digits needs to contact the object to be able to pick it up. The fact that ICMS-elicited percepts are spatially localized could then be exploited to convey information about contact location. Phantom limb sensations are presumably associated with activation in parts of the brain that used to receive signals from the limb. For an amputee or tetraplegic patient, then, one could stimulate through each electrode in turn and ask the patient where the sensation was felt on the phantom or deafferented limb. The sensor on the corresponding part of the hand could then be connected to that electrode such that anytime that sensor touched something, stimulation would be delivered through that electrode. For example, suppose electrical stimulation through electrode $N$ resulted in sensations projected to the index fingertip; the sensor on the index fingertip would then be connected to electrode $N$. Now, anytime the patient touched something with that index fingertip, he or she would experience a tactile sensation on his or her (phantom or deafferented) index fingertip (Figure 2A). In studies with rats, an analogous biomimetic approach has been shown to be effective in conveying information about stimulus location (O'Connor et al., 2013; Venkatraman and Carmena, 2011).

Information about contact pressure also plays a crucial role in our ability to grasp and manipulate objects: We need to exert enough pressure on the object to maintain grasp but not so much as to crush it. When we shake someone's hand, or touch a loved one, we want to do so with an appropriate amount 
of pressure: not too limp but not painfully hard either. Light pressure exerted on the skin results in a faint cutaneous sensation, whereas strong pressure results in a more intense one. In cortex, lowpressure skin deflections result in activation that is relatively faint and highly localized whereas highpressure skin deflections result in activation that is stronger and more widespread. To convey information about pressure biomimetically, then, one would wish to mimic the neuronal correlates of pressure perception through electrical stimulation, which can be accomplished by simply increasing the amplitude of ICMS. To convey a sensation that is appropriate to a level of pressure thus requires creating a mapping between electrical amplitude and pressure that yield percepts of equal magnitude (Figure 2b). Using such a mapping, monkeys were able to discriminate pressure as well whether the mechanical indentations were delivered to their hand or to a prosthetic hand that drove ICMS delivered to S1 in quasi-real time (Berg et al., 2013; Tabot et al., 2013). A pressure mapping could be achieved in patients by adjusting the parameters of the function relating ICMS amplitude to pressure (Figure $2 \mathrm{~b}$ ) based on results from psychophysical experiments in which patients matched the magnitude of ICMSevoked percepts to that of mechanical stimuli applied to body regions whose innervation is intact, for example the face.

Information about contact timing is also very important in grasping objects. Specifically, when we reach for an object, the reach phase ends as soon as contact with the object is established, and the grasp can be completed (Johansson and Flanagan, 2009). Information about the timing of contact events is also important in coordinating sequential movements of the fingers. In cortex, the onset and offset of contact are signaled by large transient responses in the majority of S1 neurons (Pei et al., 2009) that are relatively insensitive to other properties of the stimulus (Bensmaia et al., 2008). Such contact-related transients could be mimicked by triggering phasic ICMS pulse trains using contact sensors on the prosthetic hand (Figure 2c).

Somatosensation is a rich, multidimensional modality that relies on multiple types of receptors and conveys a wide variety of signals about objects grasped in the hand. To fully restore touch would entail restoring the various qualities of touch. One way in which somatosensory percepts with a particular quality might be elicited would be to stimulate S1 neurons that are implicated in encoding that quality. Indeed, as mentioned above, different cortical neurons seem to encode different features. For example, a population of S1 neurons respond maximally when the limb moves in a particular direction; the response of these neurons decreases as the limb trajectory deviates from this preferred direction (London and Miller, 2013). Encouragingly, electrical stimulation of neurons with similar preferred directions elicits sensations of movement in their preferred direction (Tomlinson and Miller, 2013). To convey information about limb movements, then, neuronal populations could be stimulated when the limb moves in their preferred direction. The same general principle could be used to elicit other types of sensations: Shape-related neurons could be activated to convey information about shape, texturerelated neurons could be activated to convey information about texture, etc. Again, the mapping could be achieved by asking patients to report the quality of the sensations evoked. This quality-specific feedback could coexist with the more basic information about contact location, pressure, and timing, by stimulating different neuronal structures. Indeed, feature selectivity gets stronger as one ascends the processing hierarchy. Thus, basic stimulus information (location, pressure, timing) could be conveyed by stimulating low-level cortical areas (area 3b, e.g.), which may actually encode these quantities more faithfully (Harvey et al., 2013), while more complex stimulus information (shape, motion, etc.) could be conveyed by stimulating the appropriate subpopulations of feature-selective neurons at higher stages of cortical processing (areas 1 and 2, secondary somatosensory cortex, e.g.). Given this scheme, it may actually be fortuitous that ICMS-evoked activity at low-level areas does not propagate to higher level 
cortices (Logothetis et al., 2010), probably due to its unnaturalness (see below); that way, basic signals may not significantly interfere with feature-specific ones in this scheme.

As the size and channel density of cortical interfaces increases, the resolution of the artificial percepts will increase, allowing for richer percepts conveyed with more complete coverage of the limb and higher spatial resolution. Experiments with currently available technologies will help determine the promise of the biomimetic approach as well as the limits of achievable naturalism in artificial somatosensation.

\section{Adaptation}

The idea behind the biomimetic approach is that reproducing naturalistic patterns of neuronal activity through ICMS will elicit verisimilar percepts that will be intuitive and therefore will not require the patient to learn to interpret the artificial sensations. The problem with the biomimetic approach is that electrical stimulation of neuronal tissue does not produce naturalistic patterns of neuronal activity; in fact, the effects of ICMS on neurons is ill-understood and difficult to predict. First, ICMS evokes highly synchronized activity, entrained to the pulse frequency, across large populations of neurons, a pattern of activation that does not occur in a normally functioning brain (Dinse et al., 1993; Godde et al., 2002). Second, the spatial extent of ICMS-evoked activity is difficult to estimate. On the one hand, the propagation of charge through the tissue is relatively predictable, with decay inversely proportional to the square of the distance from the electrode tip (Stoney et al., 1968; Tehovnik, 1996). The passive diffusion of charge thus predicts that the volume of activated neuronal tissue increases as the stimulation amplitude increases. On the other hand, axons have the lowest thresholds for electrical stimulation and so are preferentially excited by ICMS (Stuart et al., 1997; Tehovnik, 1996). Thus, the spatial distribution of excitation is determined in part by the pattern of axonal arborization and so varies from area to area and indeed from site to site within a single area (Histed et al., 2009; Overstreet et al., 2013).

Given the inability to elicit natural (or even predictable) patterns of neuronal activity through ICMS, one might be tempted to abandon the biomimetic strategy altogether. Indeed, it is not clear that the ICMSevoked activity will be similar enough to natural activity, under any circumstance, for that bit of similarity to be meaningful and thus yield an increment of intuitiveness. An alternative strategy consists of exploiting the ability of the nervous system to learn and adapt. The so-called 'adaptation' approach, then, involves creating a mapping between patterns of sensor activation on the prosthetic limb and patterns of ICMS to S1 that is systematic but not necessarily biomimetic. The idea is that the patient will learn the relationship between ICMS-elicited sensations and the state of the limb or the properties of grasped objects upon repeated pairings.

Animals have been shown to be able to perform tasks based on patterns of ICMS that were not designed to elicit naturalistic patterns of neuronal activation. In one such study, a monkey learned to distinguish two "virtual textures," each consisting of a different temporal pattern of ICMS applied to S1 (O'Doherty et al., 2011). While the sensory component of the task was analogous to the frequency discrimination task described above (Romo et al., 1998), this study was significant in that it was the first to combine artificial sensory feedback with online decoding of motor signals for real time control of a virtual limb. While these studies were performed in parts of S1 that respond primarily to cutaneous stimulation, analogous results were obtained in proprioceptive areas (namely area 3a)(London et al., 2008). In the studies described thus far, animals learned to discriminate small numbers of discrete patterns of ICMS. In a pair of studies, animals were shown to be able to use continuously varying ICMS signals to guide behavior. In one study, the strength of ICMS delivered to the somatosensory cortex of rats was 
modulated according to the distance of the rat's head to a water port: the closer the rat's head was to the port, the higher the ICMS pulse frequency (Thomson et al., 2013). The rat learned to locate the waterport based on this artificial signal. In an analogous study, a monkey learned to guide a cursor on a screen based on ICMS delivered across eight electrodes (Dadarlat et al., 2012)(Figure 3). In this experiment, the spatio-temporal pattern of ICMS was determined not only by the distance of the cursor from the target, but also by the location of the cursor relative to the target: Different electrodes were associated with different angles between cursor and target, and the differential activation across electrodes thus provided the animal with information about the direction in which to move the cursor. During the training phase, the ICMS was paired with visual signals carrying the same target-related information, allowing the animal to learn the task. In these studies, then, the (continuous) artificial sensory feedback guided the animal's behavior despite the fact that it did not correspond to signals normally found in the brain.

If animals can learn to interpret novel artificial sensory feedback and use it to guide behavior, why bother even attempting to mimic natural sensation? The adaptation approach is appealing because it does not require that naturalistic patterns of neuronal activation be elicited, which, as discussed above, sidesteps a significant limitation of the biomimetic approach. However, the upper limb can move in many different ways and the number of patterns of cutaneous stimulation (and their analogs on a prosthetic hand) is virtually infinite. Ideally, the artificial sensory feedback would allow the patient to track the state of the limb and convey information about objects grasped in the hand to the same degree as does natural somatosensory feedback. A major challenge for an approach based purely on adaptation is whether it can accommodate such a complex space. Indeed, a patient would have to learn to associate some sets of sensations with movements of joints, other sets of sensations with contact with objects, etc. Whether learning such a complex sensory space from scratch is possible in adulthood remains to be determined. Indeed, despite the fact that juvenile brains are designed to learn to interpret sensory signals and use them to guide motor behavior, it takes children years to learn to use their hands dexterously. The adult brain may take even longer to learn to interpret such complex and completely novel sensory feedback or may not be able to learn it at all. Even if the artificial feedback is learnable, patients may not have the patience to do so and may thus reject this highly invasive restorative strategy. One way to get around the problem of scalability is to design mappings between limb state and ICMS in a principled way, such that the artificial feedback is optimally informative given the limitations inherent to ICMS (cf. Daly et al., 2012). However, such approaches are still in their infancy and their benefits to guiding behavior have yet to be explored.

\section{Contributions of biomimicry and adaptation}

The biomimetic and adaptation approaches are not mutually exclusive and indeed are complementary. Studies with biomimetic ICMS have demonstrated that, for simple stimulus quantities, like location or pressure, animals can generalize instantaneously from natural to artificial feedback. However, the evoked sensations are almost certainly not naturalistic and it is not clear whether biomimetic strategies to elicit more complex sensations will be successful. The ability to adapt to novel sensory input is thus likely to help sharpen biomimetically-generated sensations and make them more verisimilar. Adaptation may also allow for a more complex artificial sensory space in which patients learn to use information that is available but not explicitly written in the biomimetic encoding algorithms. However, to the extent that the initial maps are biomimetic, the patient will have less to learn and the adaptation process will consist in refining sensory representations rather than constructing them from scratch. 
A compelling example of the interplay between biomimicry and adaptation is in the progression of embodiment of prosthetic limbs demonstrated in patients with targeted muscle reinervation. These patients undergo surgery to reroute nerves that once innervated a severed limb into intact skin and muscles. When this newly innervated skin is touched, these individuals experience tactile sensations that are projected to the phantom limb. In a study inspired by the rubber hand illusion, tactile stimuli were repeatedly applied to the newly innervated skin, which were experienced somewhere on the phantom; these stimuli were paired with the visual experience of touches applied to a location on the prosthetic limb that corresponded to the felt location of the tactile stimuli (Marasco et al., 2011). For example, if stimulation of a patch of skin on the chest elicited a sensation on the forearm, touches on that patch of skin were paired with touches on the prosthetic forearm (which of course were seen but not felt). The repeated pairing caused the tactile sensations to be experienced on the prosthetic limb rather than on the phantom limb. Such a migration is likely to also be observed when vision and artificial somatosensation result in spatially and temporally congruent percepts (Shokur et al., 2013).

\section{Plasticity}

Somatosensory cortex has been shown to change adaptively when the pattern of afferentation changes. For example, when a digit is stimulated more than its neighbors, its cortical magnification increases (Elbert et al., 1995; Jenkins et al., 1990). Changes in cortical magnification are also observed in medical conditions when some body parts are overused, such as writer's cramp and carpal tunnel (Nelson et al., 2009; Tecchio et al., 2002). Similarly, when a digit is severed, the cortical representation starts to respond to that of adjacent digits (Merzenich et al., 1984; Weiss et al., 2000a). If the whole limb is severed, the deafferented cortex becomes responsive to other body parts, determined in part by the proximity of their cortical representation and the degree to which they are used during activities of daily living (Karl et al., 2001; Makin et al., 2013). This progressive reorganization of cortex is thought to underlie the perceptual shrinking of the phantom limb over time (Flor et al., 1995). The receptive fields of S1 neurons can even adapt to compensate for the surgical rotation of patches of skin (Rosselet et al., 2008).

If the parts of the brain that represent the arm and hand can change so dramatically, can we still hope to exploit their topographical organization even years after the injury? The outlook is not so bleak when we examine the extent and dynamics of cortical plasticity. First, the functional significance of somatotopically inappropriate activations has yet to be established: Indeed, body regions that "invade" the deafferented cortex are no more sensitive than are their counterparts on the intact side, which do not benefit from the additional cortical representation (Grusser et al., 2001). Thus, the invading representations do not seem to reafferent cortex in a demonstrably functional way. Second, changes in topography can occur within hours or days after amputation (Borsook et al., 1998; Weiss et al., 2000b) and even immediately after peripheral anesthesia of fingers in intact hands (Waberski et al., 2007; Waberski et al., 2003). Conversely, it is possible to instantly reverse the encroachment of lip into the digit representation by anesthetizing the amputated stump (Birbaumer et al., 1997). Changes in somatotopy can thus be observed on time scales that are too fast to reflect significant neuronal rewiring and can be just as rapidly reversed (in the case of the anesthesia experiments). Third, changes in topography are observed in thalamus (Davis et al., 1998; Lenz and Byl, 1999; Lenz et al., 1998) and may in fact be attributable to plastic changes in cuneate nucleus (Kambi et al., 2014). Importantly, stimulation of regions in thalamus that respond to the limb stump elicits sensations in the phantom digits, not the stump (Davis et al., 1998). Thus, the remapping of S1 does not necessarily reflect changed circuitry in S1. Fourth, some amputees develop distinct patches of skin on their limb stump, stimulation of which results in a percept projected to individual digits on the phantom hand and activation in the 
corresponding digit representation in S1 (Bjorkman et al., 2012). As might be expected, then, electrical stimulation of deafferented regions of $\mathrm{S} 1$ in human amputees evokes sensations on the phantom limb rather than on the invading body regions (Ojemann and Silbergeld, 1995; Woolsey et al., 1979). Fifth, reorganization of S1 reverts back to its pre-amputation somatotopy after a hand transplantation (Frey et al., 2008). Reafferentation of S1 thus seems to restore its original topography, suggesting that invading signals from other body regions may simply reflect the unmasking of lateral connections and not major structural and functional changes in deafferented cortex (although some abnormal competition between the original and reafferented body region may linger, see Farne et al., 2002). The body maps in S1 are interconnected with a variety of other spatial representations distributed throughout the nervous system, which might explain why they are largely immutable in adulthood. The evidence suggests, then, that deafferented cortex has not dramatically changed structurally or functionally as a consequence of deafferentation and is primed for reafferentation. However, most of the assays described above are rather crude and do not probe how the functional properties of deafferented neurons may have changed as a result of injury. Instrumenting human patients with cortical interfaces with penetrating electrodes will help fill this gap by providing an opportunity to investigate the perceptual consequences of selectively stimulating small neuronal populations.

\section{Reliability and safety}

For ICMS to be a viable approach for conveying somatosensory feedback, it must be both reliable and safe. Unfortunately, chronically implanted electrode arrays are not sufficiently robust to last in the brain for decades. Indeed, the electrodes degrade over time (Kane et al., 2013; Prasad et al., 2012) and electrode arrays are susceptible to outright failure, most often due to connector issues (Barrese et al., 2013; Simeral et al., 2011). Given the highly invasive surgery required for implantation and the fact that defective arrays cannot be replaced, intracortical interfaces are thus not ready to be deployed clinically. Some evidence suggests that chronic ICMS can cause neuronal loss (McCreery et al., 2010), but it is unclear whether this loss has any functional consequences (Chen et al., 2014; Parker et al., 2011). Furthermore, chronic ICMS does not seem to cause additional damage to the electrodes or the tissue (Chen et al., 2014). Thus, it does not seem as though the inclusion of chronic ICMS will pose a significant additional challenge to the clinical deployment of cortical interfaces. Nonetheless, interface technology must improve before it is ready for widespread use.

\section{Conclusions}

The development of anthropomorphic robotic arms and of algorithms to decode motor intention based on signals from the brain has led to remarkable demonstrations of human patients controlling robotic arms by thought (Collinger et al., 2013; Hochberg et al., 2012). However, this technology will likely not transition into the clinic until somatosensory feedback is incorporated. Indeed, controlling an arm without somatosensation is challenging even with an intact motor system. While patients with cortical interfaces can use the arms to perform simple tasks, their movements are slow and clumsy, and may not justify the dangerous and invasive surgery brain-machine interfaces require. Restoring the senses of touch and proprioception through ICMS in S1 is a promising approach but one whose development is still in its infancy. Studies on biomimetic sensory feedback will show us how verisimilar artificial percepts can be, and studies on adaptation will teach us how much we can adapt to artificial sensory input. Soon, then, a human being will touch an object through a robotic hand, and science will have once again caught up with science fiction. 
Barrese, J. C., et al., 2013. Failure mode analysis of silicon-based intracortical microelectrode arrays in non-human primates. J Neural Eng. 10, 066014.

Bensmaia, S. J., et al., 2008. The representation of stimulus orientation in the early stages of somatosensory processing. J Neurosci. 28, 776-86.

Bensmaia, S. J., Tillery, S. H., Tactile feedback from the hand. In: R. Balasubramaniam, et al., (Eds.), The Human Hand: A Source of Inspiration for Robotic Hands. Springer, New York, 2013.

Berg, J. A., et al., 2013. Behavioral demonstration of a somatosensory neuroprosthesis. IEEE Trans Neural Syst Rehabil Eng. 21, 500-7.

Biddiss, E., et al., 2007. Consumer design priorities for upper limb prosthetics. Disabil Rehabil Assist Technol. 2, 346-57.

Birbaumer, N., et al., 1997. Effects of regional anesthesia on phantom limb pain are mirrored in changes in cortical reorganization. J Neurosci. 17, 5503-8.

Bjorkman, A., et al., 2012. Phantom digit somatotopy: a functional magnetic resonance imaging study in forearm amputees. Eur J Neurosci. 36, 2098-106.

Björnsdotter, M., et al., 2009. Somatotopic organization of gentle touch processing in the posterior insular cortex. The Journal of Neuroscience. 29, 9314-9320.

Borsook, D., et al., 1998. Acute plasticity in the human somatosensory cortex following amputation. Neuroreport. 9, 1013-7.

Botvinick, M., Cohen, J., 1998. Rubber hands 'feel' touch that eyes see. Nature. 391, 756.

Brochier, T., et al., 1999. The effects of muscimol inactivation of small regions of motor and somatosensory cortex on independent finger movements and force control in the precision grip. Exp Brain Res. 128, 31-40.

Chen, K. H., et al., 2014. The effect of chronic intracortical microstimulation on the electrode-tissue interface. J Neural Eng. 11, 026004.

Collinger, J. L., et al., 2013. High-performance neuroprosthetic control by an individual with tetraplegia. Lancet. 381, 557-64.

Dadarlat, M. C., et al., Multisensory integration of vision and intracortical microstimulation for sensory substitution and augmentation. In: S. f. Neuroscience, (Ed.), Society for Neuroscience. Society for Neuroscience, New Orleans, LA, 2012.

Daly, J., et al., 2012. Optimal space-time precoding of artificial sensory feedback through mutichannel microstimulation in bi-directional brain-machine interfaces. J Neural Eng. 9, 065004.

Davis, K. D., et al., 1998. Phantom sensations generated by thalamic microstimulation. Nature. 391, 3857.

Dinse, H. R., et al., 1993. Alterations in correlated activity parallel ICMS-induced representational plasticity. Neuroreport. 5, 173-176.

Edin, B. B., 1990. Finger joint movement sensitivity of non-cutaneous mechanoreceptor afferents in the human radial nerve. Exp Brain Res. 82, 417-22.

Edin, B. B., 2004. Quantitative analyses of dynamic strain sensitivity in human skin mechanoreceptors. J Neurophysiol. 92, 3233-43.

Elbert, T., et al., 1995. Increased cortical representation of the fingers of the left hand in string players. Science. 270, 305-307.

Farne, A., et al., 2002. Face or hand, not both: perceptual correlates of reafferentation in a former amputee. Curr Biol. 12, 1342-6.

Flor, H., et al., 1995. Phantom-limb pain as a perceptual correlate of cortical reorganization following arm amputation. Nature. 375, 482-4.

Frey, S. H., et al., 2008. Chronically deafferented sensory cortex recovers a grossly typical organization after allogenic hand transplantation. Curr Biol. 18, 1530-4. 
Gazzola, V., et al., 2012. Primary somatosensory cortex discriminates affective significance in social touch. Proceedings of the National Academy of Sciences. 109, E1657-E1666.

Godde, B., et al., 2002. Plasticity of orientation preference maps in the visual cortex of adult cats. Proceedings of the National Academy of Sciences. 99, 6352-6357.

Gordon, J., et al., 1995. Impairments of reaching movements in patients without proprioception. I. Spatial errors. J Neurophysiol. 73, 347-60.

Grusser, S. M., et al., 2001. The relationship of perceptual phenomena and cortical reorganization in upper extremity amputees. Neuroscience. 102, 263-72.

Harvey, M. A., et al., 2013. Multiplexing stimulus information through rate and temporal codes in primate somatosensory cortex. PLoS Biol. 11, e1001558.

Hertenstein, M. J., et al., 2009. The communication of emotion via touch. Emotion. 9, 566.

Histed, M. H., et al., 2009. Direct activation of sparse, distributed populations of cortical neurons by electrical microstimulation. Neuron. 63, 508-522.

Hochberg, L. R., et al., 2012. Reach and grasp by people with tetraplegia using a neurally controlled robotic arm. Nature. 485, 372-5.

Jenkins, W. M., et al., 1990. Functional reorganization of primary somatosensory cortex in adult owl monkeys after behaviorally controlled tactile stimulation. Journal of neurophysiology. 63, 82104.

Johansson, R. S., Flanagan, J. R., 2009. Coding and use of tactile signals from the fingertips in object manipulation tasks. Nat Rev Neurosci. 10, 345-59.

Johnson, L. A., et al., 2013. Direct electrical stimulation of the somatosensory cortex in humans using electrocorticography electrodes: a qualitative and quantitative report. J Neural Eng. 10, 036021.

Kambi, N., et al., 2014. Large-scale reorganization of the somatosensory cortex following spinal cord injuries is due to brainstem plasticity. Nat Commun. 5, 3602.

Kane, S., et al., 2013. Electrical performance of penetrating microelectrodes chronically implanted in cat cortex. IEEE Trans Biomed Eng.

Karl, A., et al., 2001. Reorganization of motor and somatosensory cortex in upper extremity amputees with phantom limb pain. The Journal of Neuroscience. 21, 3609-3618.

Keysers, C., et al., 2010. Somatosensation in social perception. Nature Reviews Neuroscience. 11, 417428.

Kringelbach, M. L., 2005. The human orbitofrontal cortex: linking reward to hedonic experience. Nature Reviews Neuroscience. 6, 691-702.

Lenz, F. A., Byl, N. N., 1999. Reorganization in the cutaneous core of the human thalamic principal somatic sensory nucleus (Ventral caudal) in patients with dystonia. J Neurophysiol. 82, 3204-12.

Lenz, F. A., et al., 1998. Neuronal activity in the region of the thalamic principal sensory nucleus (ventralis caudalis) in patients with pain following amputations. Neuroscience. 86, 1065-81.

Logothetis, N. K., et al., 2010. The effects of electrical microstimulation on cortical signal propagation. Nature Neuroscience. 13, 1283-91.

Löken, L. S., et al., 2009. Coding of pleasant touch by unmyelinated afferents in humans. Nature neuroscience. $12,547-548$.

London, B. M., et al., 2008. Electrical stimulation of the proprioceptive cortex (area 3a) used to instruct a behaving monkey. IEEE Trans Neural Syst Rehabil Eng. 16, 32-6.

London, B. M., Miller, L. E., 2013. Responses of somatosensory area 2 neurons to actively and passively generated limb movements. J Neurophysiol. 109, 1505-13.

Makin, T. R., et al., 2013. Phantom pain is associated with preserved structure and function in the former hand area. Nature communications. 4, 1570.

Marasco, P. D., et al., 2011. Robotic touch shifts perception of embodiment to a prosthesis in targeted reinnervation amputees. Brain. 134, 747-58. 
Marsden, C., et al., 1984. The use of peripheral feedback in the control of movement. Trends in Neurosciences. 7, 253-257.

McCreery, D., et al., 2010. Neuronal loss due to prolonged controlled-current stimulation with chronically implanted microelectrodes in the cat cerebral cortex. J Neural Eng. 7, 036005.

Merzenich, M. M., et al., 1984. Somatosensory cortical map changes following digit amputation in adult monkeys. Journal of comparative neurology. 224, 591-605.

Nelson, A. J., et al., 2009. Digit-specific aberrations in the primary somatosensory cortex in Writer's cramp. Ann Neurol. 66, 146-54.

O'Connor, D. H., et al., 2013. Neural coding during active somatosensation revealed using illusory touch. Nat Neurosci. 16, 958-65.

O'Doherty, J. E., et al., 2011. Active tactile exploration using a brain-machine-brain interface. Nature. 479, 228-31.

Ojemann, J. G., Silbergeld, D. L., 1995. Cortical stimulation mapping of phantom limb rolandic cortex. Case report. J Neurosurg. 82, 641-4.

Overstreet, C., et al., 2013. Computational modeling of direct neuronal recruitment during intracortical microstimulation in somatosensory cortex. Journal of neural engineering. 10, 066016.

Parker, R. A., et al., The functional consequences of chronic, physiologically effective intracortical microstimulation. In: J. Schouenborg, et al., (Eds.), Progress in Brain Research. Elsevier, 2011, pp. 145-165.

Pei, Y. C., et al., 2009. Convergence of submodality-specific input onto neurons in primary somatosensory cortex. J Neurophysiol. 102, 1843-53.

Pei, Y. C., et al., 2010. Shape invariant coding of motion direction in somatosensory cortex. PLoS Biol. 8, e1000305.

Pei, Y. C., et al., 2011. Neural mechanisms of tactile motion integration in somatosensory cortex. Neuron. 69, 536-47.

Penfield, W., Boldrey, E., 1937. Somatic motor and sensory representation in the cerebral cortex of man as studied by electrical stimulation. Brain 60, 389-443.

Prasad, A., et al., 2012. Comprehensive characterization and failure modes of tungsten microwire arrays in chronic neural implants. J Neural Eng. 9, 056015.

Ramachandran, V. S., Hirstein, W., 1998. The perception of phantom limbs. The D. O. Hebb lecture. Brain. 121 ( Pt 9), 1603-30.

Rolls, E. T., et al., 2003. Representations of pleasant and painful touch in the human orbitofrontal and cingulate cortices. Cerebral Cortex. 13, 308-317.

Romo, R., et al., 2000. Sensing without touching: Psychophysical performance based on cortical microstimulation. Neuron. 26, 273-278.

Romo, R., et al., 1998. Somatosensory discrimination based on cortical microstimulation. Nature. 392, 387-390.

Rosselet, C., et al., 2008. Experience-dependent changes in spatiotemporal properties of cutaneous inputs remodel somatosensory cortical maps following skin flap rotation. Eur J Neurosci. 27, 1245-60.

Sainburg, R. L., et al., 1995. Control of limb dynamics in normal subjects and patients without proprioception. J Neurophysiol. 73, 820-35.

Sainburg, R. L., et al., 1993. Loss of proprioception produces deficits in interjoint coordination. J Neurophysiol. 70, 2136-47.

Sherman, R. A., 1989. Stump and phantom limb pain. Neurol Clin. 7, 249-64.

Shokur, S., et al., 2013. Expanding the primate body schema in sensorimotor cortex by virtual touches of an avatar. Proceedings of the National Academy of Sciences. 
Simeral, J. D., et al., Some preliminary longitudinal findings from five trial participants using the BrainGate neural interface system. Society for Neuroscience, Vol. 142.04. Society for Neuroscience, San Diego, CA, 2011.

Stoney, S., et al., 1968. Excitation of pyramidal tract cells by intracortical microstimulation: effective extent of stimulating current. Journal of neurophysiology. 31, 659-669.

Stuart, G., et al., 1997. Action potential initiation and backpropagation in neurons of the mammalian CNS. Trends in neurosciences. 20, 125-131.

Tabot, G. A., et al., 2013. Restoring the sense of touch with a prosthetic hand through a brain interface. Proc Natl Acad Sci U S A. 110, 18279-84.

Tecchio, F., et al., 2002. Carpal tunnel syndrome modifies sensory hand cortical somatotopy: a MEG study. Human brain mapping. 17, 28-36.

Tehovnik, E. J., 1996. Electrical stimulation of neural tissue to evoke behavioral responses. Journal of neuroscience methods. 65, 1-17.

Thomson, E. E., et al., 2013. Perceiving invisible light through a somatosensory cortical prosthesis. Nat Commun. 4, 1482.

Tomlinson, T., Miller, L. E., Multi-electrode stimulation in somatosensory are 2 induces a natural sensation of limb movement. Society for Neuroscience. Society for Neuroscience, San Diego, CA, 2013.

Tsakiris, M., Haggard, P., 2005. The rubber hand illusion revisited: visuotactile integration and selfattribution. J Exp Psychol Hum Percept Perform. 31, 80-91.

Venkatraman, S., Carmena, J. M., 2011. Active sensing of target location encoded by cortical microstimulation. IEEE Trans Neural Syst Rehabil Eng. 19, 317-24.

Waberski, T. D., et al., 2007. Short-term cortical reorganization by deafferentation of the contralateral sensory cortex. Neuroreport. 18, 1199-203.

Waberski, T. D., et al., 2003. Immediate cortical reorganization after local anesthetic block of the thumb: source localization of somatosensory evoked potentials in human subjects. Neurosci Lett. 347, 151-4.

Weiss, T., et al., 2000a. Rapid functional plasticity of the somatosensory cortex after finger amputation. Experimental brain research. 134, 199-203.

Weiss, T., et al., 2000b. Rapid functional plasticity of the somatosensory cortex after finger amputation. Exp Brain Res. 134, 199-203.

Woolsey, C. N., et al., 1979. Localization in somatic sensory and motor areas of human cerebral cortex as determined by direct recording of evoked potentials and electrical stimulation. J Neurosurg. 51, 476-506.

Wright, T. W., et al., 1995. Prosthetic usage in major upper extremity amputations. J Hand Surg Am. 20, 619-22. 

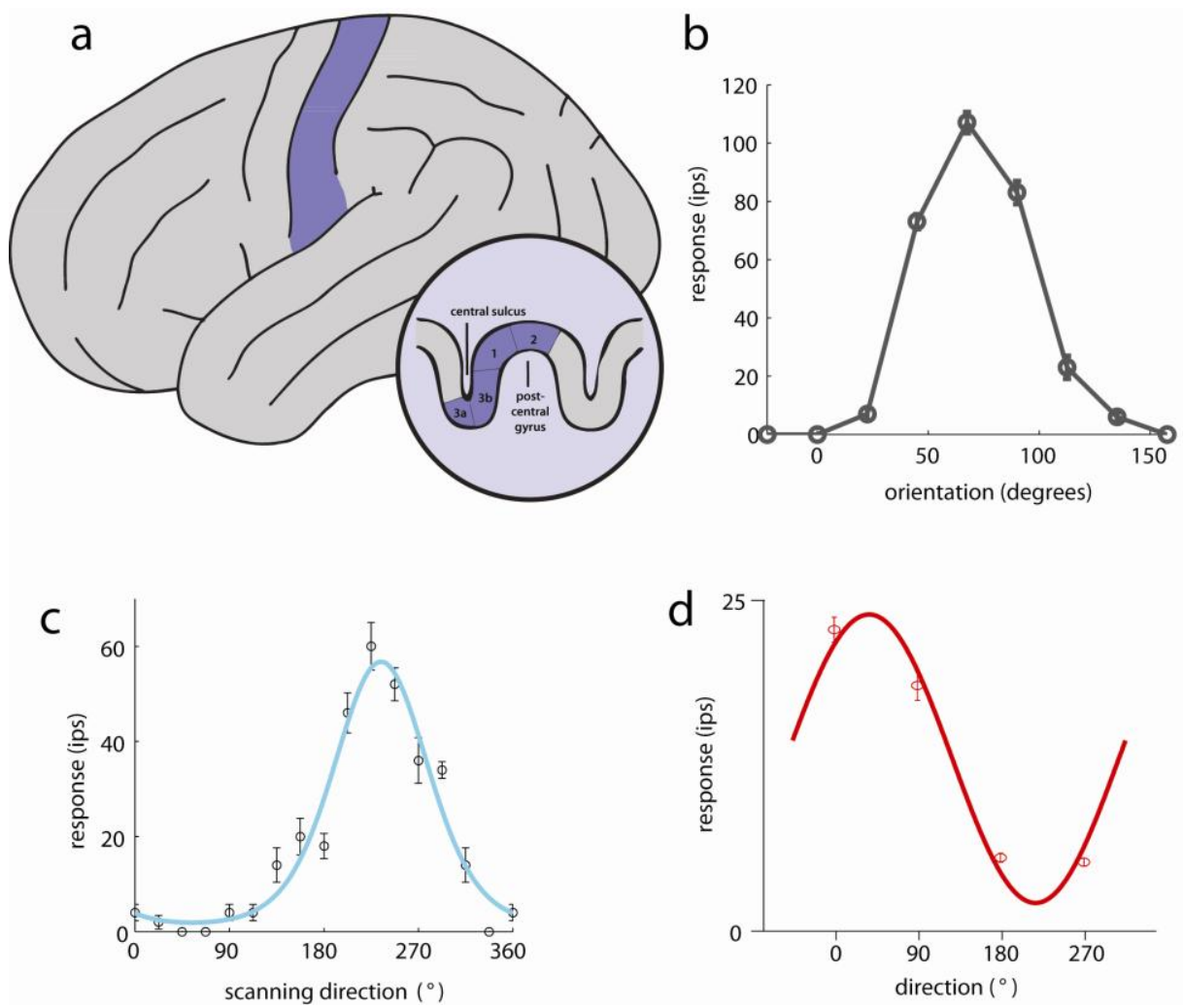

Figure 1. Primary somatosensory cortex | a. Primary somatosensory cortex is located in anterior parietal cortex, on the postcentral gyrus, and comprises four modules, Brodmann's areas 3a, 3b, 1 and 2. Neurons in area 3a respond primarily to joint movements, neurons in areas $3 \mathrm{~b}$ and 1 respond to cutaneous stimulation, and neurons in area 2 exhibit both proprioceptive and cutaneous responses. b. Orientation-selective neuron in area $3 \mathrm{~b}$ : This neuron responds preferentially when an edge whose orientation is parallel to the long axis of the digit is indented into the fingertip (Bensmaia et al., 2008). c. Directionselective (cutaneous) neuron in area 1: This neuron responds preferentially when a stimulus (in this case an edge) is scanned across the fingertip in the distal-to-proximal direction (Pei et al., 2010). d. Direction-selective (proprioceptive) neuron in area 2: This neuron responds preferentially to a limb deflection in the proximal-to-distal direction (towards the body)(London and Miller, 2013). 

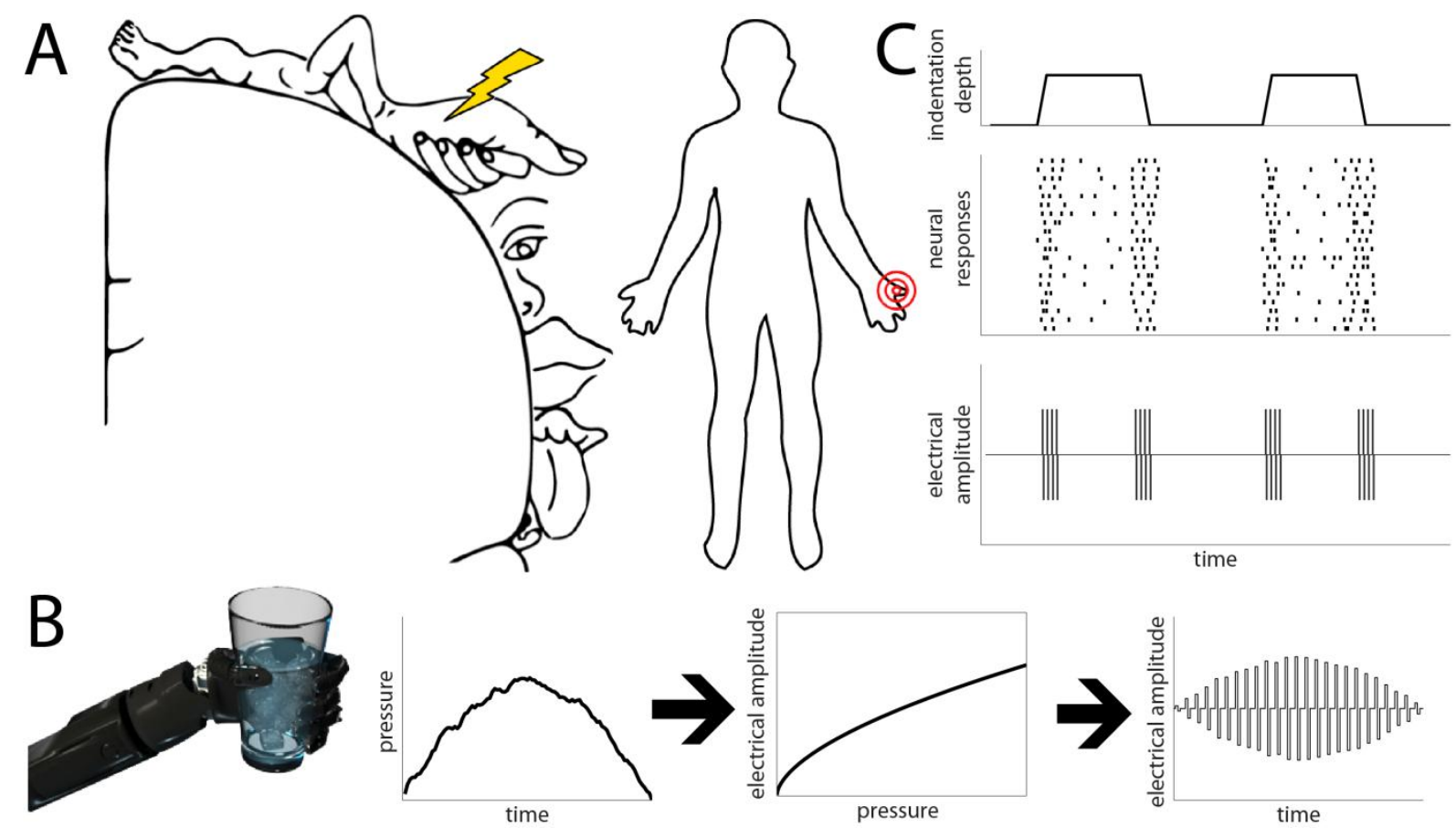

Figure 2. Biomimetic approach | a. Contact location: Stimulating a local population of S1 neurons elicits a percept that is projected to the receptive field of those neurons on the skin. b. Contact pressure: Pressure signals from the sensors on the hand can be converted into electrical pulse trains that elicit sensations of appropriate sensory magnitude. c. Timing of contact events: The top trace shows the temporal profile of two indentations into the skin. The middle trace, a representation of the evoked response, and the bottom trace the biomimetic pulse train that signals contact. With an intact arm and nervous system, contact events are signaled by strong transient responses in almost all S1 neurons. These transients can be replicated by delivering phasic pulse trains triggered at the onset and offset of contact. Note that, a force-related signal (not shown) would be superimposed on the contact-related one. 

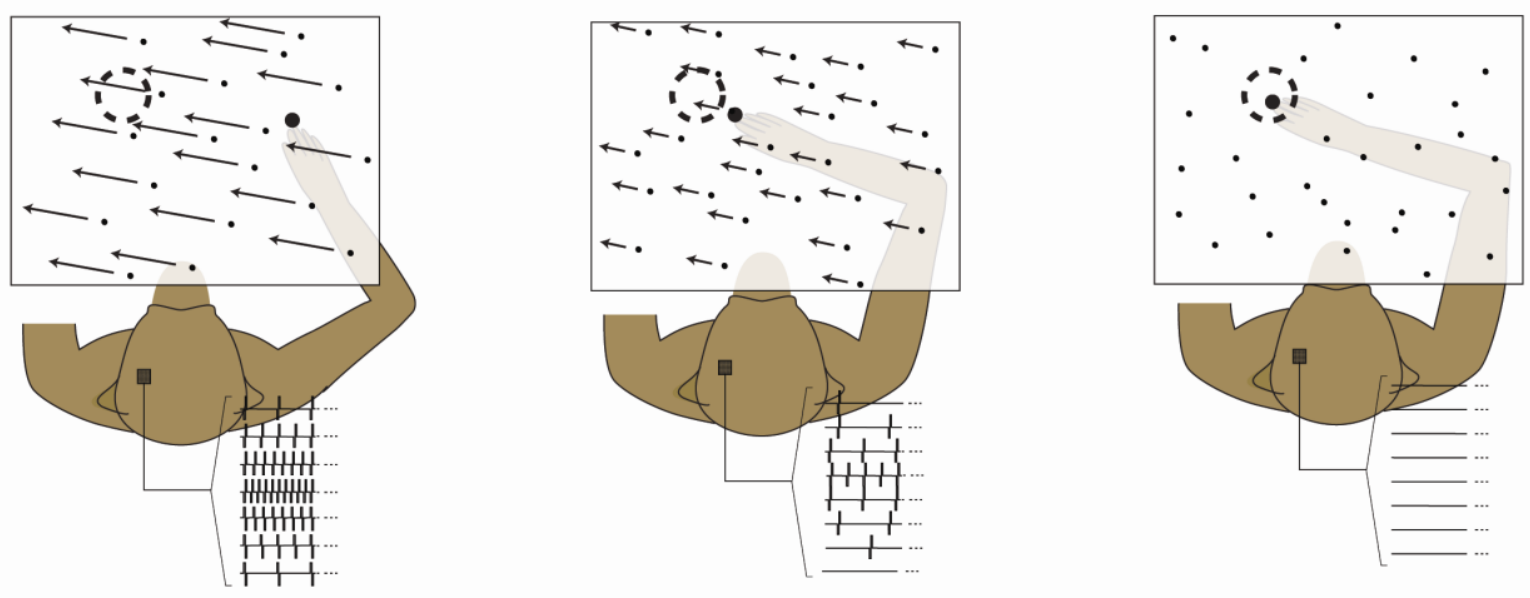

Figure 3. Example of the adaptation approach | The direction and speed of a random dot stereogram indicate to the animal the relative location and distance of the target, respectively. The patterning in and strength of ICMS convey redundant information about the relative location and distance of the target, respectively. The animal is trained with visual stimulation and ICMS but eventually can do the task based on artificial feedback alone (Dadarlat et al., 2012). 